\title{
Letter
}

\section{COVID-19 in Patients With Connective Tissue Disease-related Interstitial Lung Disease}

To the Editor:

Infection with the coronavirus disease 2019 (COVID-19) caused by the severe acute respiratory syndrome coronavirus 2 (SARS-CoV-2) manifests in a myriad of ways, ranging from asymptomatic disease to pneumonia and acute respiratory distress syndrome. Advanced age and underlying cardiovascular/pulmonary conditions appear to increase the risk for COVID-19 complications $s^{1,2}$. Patients with connective tissue disease-related interstitial lung disease (CTD-ILD) may represent a vulnerable patient population for COVID-19 given their diminished pulmonary reserve. To our knowledge, there are no prospective data reporting outcomes of SARS-CoV-2 infection in patients with CTD-ILD. We herein present all known cases $(n=4)$ of COVID-19 in patients with CTD-ILD at the University of California Los Angeles (UCLA) between January 2020 and August 2020.

Ethics approval was not obtained for this study. The UCLA Institutional Review Board does not consider cases studies to be research as defined by federal regulations. We obtained all patients' written informed consent to publish the material.

Case 1: Systemic sclerosis-related ILD. EL is a 55-year-old Latina female with pulmonary hypertension and progressive systemic sclerosis-related (SSc) ILD receiving mycophenolate mofetil (MMF) $1 \mathrm{~g}$ twice per day for 5 years (Table 1). In early April 2020, the patient developed fevers, malaise, sore throat, cough, and dyspnea after exposure to her son-in-law who tested positive by nasopharyngeal swab for SARS-CoV-2. She self-quarantined, discontinued MMF, and received supportive care at home. Her symptoms resolved after 3 weeks, and she required no increase in her baseline supplemental oxygen requirements of 3-5 L. On April 24, she tested positive for IgG anti- bodies to SARS-CoV-2. A high-resolution computed tomography (HRCT) scan of the chest demonstrated overall unchanged moderate to severe ILD without evidence of ground glass opacity or consolidations. MMF was reinitiated, and the patient has experienced no subsequent sequela of COVID-19 in the 3 months following her illness.

Case 2: SSc-ILD. RD is a 66-year-old Latina female with obesity, diabetes mellitus, and SSc-ILD taking mycophenolic acid (MPA) $760 \mathrm{mg}$ twice per day for 1 year (Table 1). In early March 2020, she accompanied her friend on a 15-h flight and shared a room with her on a 10-day cruise. On Day 4 of the cruise, they both developed fevers, myalgias, nausea, diarrhea, cough, loss of taste, and dyspnea. Upon disembarking from the cruise, she self-quarantined, discontinued MPA, and received support care at home. Her oxygen saturation remained within normal limits on ambient air. Her symptoms resolved after 3 weeks. Her friend who did not have CTD-ILD and was not receiving immunosuppression developed worsening dyspnea, prompting intubation in the emergency department, where she tested positive for SARS-CoV-2 by nasopharyngeal swab and died 5 days later. Two months after her initial illness, RD tested positive for IgG antibodies to SARS-CoV-2. MPA was reinitiated, and the patient has experienced no subsequent sequela of COVID-19 in the 4 months following her illness.

Case 3: Rheumatoid arthritis-ILD. SP is a 70-year-old white female with rheumatoid arthritis (RA) and mild ILD taking azathioprine (AZA) $50 \mathrm{mg}$ daily for 3 years (Table 1, Figure 1A). On March 16, 2020, the patient developed fevers, progressive cough, dyspnea, malaise, and nausea after her husband returned from a ski trip to Vail, Colorado, USA. Both she and her husband tested positive for SARS-CoV-2 by nasopharyngeal swab. She self-quarantined and discontinued AZA (Table 1). On Day 15, her dyspnea worsened, and she was admitted to the hospital for hypoxia. Her labs were notable for the following: white blood cell count $4.0 \times 103 / \mathrm{uL}$, lymphocyte

Table 1. Baseline characteristics of patients with CTD-ILD prior to COVID-19 infection.

\begin{tabular}{lcccc}
\hline & $\begin{array}{c}\text { Case 1 (EL), } \\
\text { SSc-ILD }\end{array}$ & $\begin{array}{c}\text { Case 2 (RD), } \\
\text { SSc-ILD }\end{array}$ & $\begin{array}{c}\text { Case 3 (SP), } \\
\text { RA-ILD }\end{array}$ & $\begin{array}{c}\text { Case 4 (MF), } \\
\text { SSc-ILD }\end{array}$ \\
\hline Age, yrs & 55 & 66 & 70 & 56 \\
Sex & Female & Female & Female & Female \\
Race/ethnicity & Latina & Latina & White & White \\
ILD duration, yrs & 5 & 7 & 4 & 12 \\
Radiographic ILD pattern & UIP & NSIP & UIP & UIP \\
Autoantibodies & ANA, Scl-70 & ANA, Scl-70 & ANA, CCP & ANA \\
FVC\% predicted & 52 & 65 & 150 & 72 \\
$\begin{array}{l}\text { DLCO\% predicted } \\
\text { TLC\% predicted }\end{array}$ & 12 & 53 & 74 & 43 \\
Immunomodulatory & 44 & 66 & 126 & 66 \\
treatment and dosage & MMF 1 g BID & MPA 760 mg BID & AZA* & MMF 1 g BID \\
$\begin{array}{l}\text { Duration of immunomodulatory } \\
\text { treatment, yrs }\end{array}$ & & & 50 mg daily & \\
\hline
\end{tabular}

* This patient also received rituximab $1000 \mathrm{mg}$ intravenously on Day 0 and Day 15 , five months prior to COVID-19. ANA: antinuclear antibodies; AZA: azathioprine; CCP: cyclic citrullinated peptide; COVID-19: coronavirus disease 2019; CTD: connective tissue disease; DLCO: diffusing lung capacity for carbon monoxide; FVC: forced vital capacity; ILD: interstitial lung disease; MMF: mycophenolate mofetil; MPA: mycophenolic acid; NSIP: nonspecific interstitial pneumonia; RA: rheumatoid arthritis; SSc: systemic sclerosis; TLC: total lung capacity; UIP: usual interstitial pneumonia. 
A

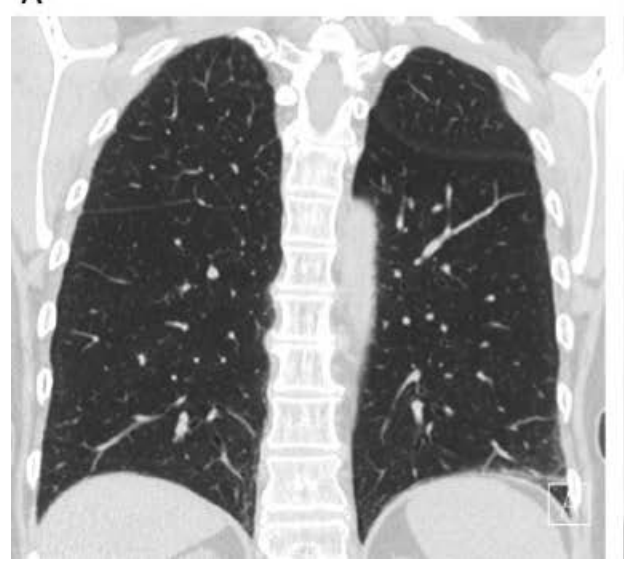

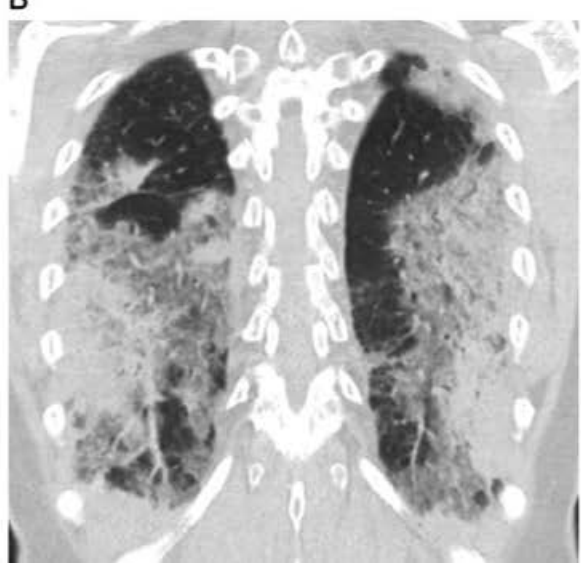

C

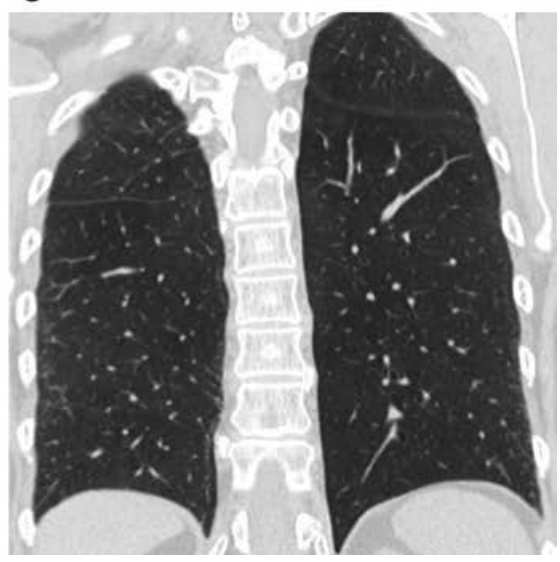

Figure 1. Serial axial HRCT images of patient SP (case 3). (A) Obtained June 9, 2019 (10 months prior to COVID-19 infection), demonstrating lower lung predominant subtle subpleural reticulation and textured ground glass attenuation with mild architectural distortion consistent with early CTD-ILD. (B) Obtained during hospitalization for COVID-19 on April 4, 2020, demonstrating mid to lower lung predominant coalescent air space consolidation and ground glass attenuation consistent with COVID-19 pneumonia. (C) Obtained 3 months after hospitalization on July 7, 2020, demonstrating interval resolution of coalescent air space consolidation and ground glass attenuation in both lungs. COVID-19: coronavirus disease 2019; CTD-ILD: connective tissue diseaserelated interstitial lung disease; HRCT: high-resolution computed tomography.

count $0.4 \times 103 / \mathrm{uL}$, neutrophil count $3.4 \times 103 / \mathrm{uL}$, ferritin $1960 \mathrm{ng} / \mathrm{mL}$, C-reactive protein (CRP) $11 \mathrm{mg} / \mathrm{dL}$, and erythrocyte sedimentation rate (ESR) $64 \mathrm{~mm} / \mathrm{h}$. Her HRCT demonstrated evidence of COVID-19 pneumonia (Figure 1B). On Day 2 of hospitalization, her oxygen requirements and inflammatory markers increased markedly, suggesting the evolution of cytokine storm syndrome (CSS). She was promptly administered tocilizumab (TCZ) $4 \mathrm{mg} / \mathrm{kg}$ intravenously. By Day 8 , her oxygen requirements returned to baseline and her inflammatory markers normalized. In the 3 months since her initial hospitalization for COVID-19, she has fully recovered, and her HRCT demonstrates complete resolution of COVID-19 pneumonia (Figure 1C).

Case 4: SSC-ILD. MF is a 56-year-old white female with SSc-ILD receiving MMF $1 \mathrm{~g}$ twice per day for 4 years (Table 1). In late July 2020, the patient developed fevers, malaise, and fatigue after exposure to her son, who tested positive by nasopharyngeal swab for SARS-CoV-2. She tested positive for SARS-CoV-2 by nasopharyngeal swab, self-quarantined, discontinued MMF, and received supportive care at home. After 2 weeks, her symptoms resolved, and she reported no change in her respiratory status. At that time, her laboratory tests were notable for a white blood cell count $6.2 \times 103 / \mathrm{uL}$, lymphocyte count $1.8 \times 103 / \mathrm{uL}$, neutrophil count $3.5 \times 103 / \mathrm{uL}$, ferritin $220 \mathrm{ng} / \mathrm{mL}$, CRP $0.4 \mathrm{mg} / \mathrm{dL}$, and ESR $42 \mathrm{~mm} / \mathrm{h}$. MMF was reinitiated, and the patient has experienced no subsequent sequela of COVID-19 in the 4 weeks following her illness.

The present cases highlight the diverse spectrum of disease manifestations and outcomes of patients with CTD-ILD and COVID-19. The 3 patients with SSc who had more severe ILD and were taking background immunomodulatory therapy with MMF/MPA had a relatively mild disease course and no exacerbation of their underlying ILD. In contrast, the patient with mild RA-ILD on low-dose AZA therapy developed pneumonia and CSS. The reasons for these disparate outcomes are unknown. It is conceivable that immunomodulator agents with different mechanisms of action may have varying success in curtailing the intensity of inflammatory response to SARS-CoV-2 infection. For instance, studies have demonstrated that MPA inhibits numerous cytokines implicated as key mediators of CSS including interferon- $\gamma$, interleukin (IL)-4, IL-13, IL-8, colony-stimulating factors, and tumor necrosis factor- $\alpha^{3}$. A single case study demonstrated that a patient with SSc-ILD undergoing treatment with TCZ also experienced a milder course of COVID-194. In a small, retrospective study of 28 ILD patients with COVID-19 (10 of whom had CTD), increased levels of IL-6, IL-1 $\beta$, and IL-2R were associated with worse clinical outcomes; however, information regarding the underlying CTD and background ILD-targeted therapy was not provided 5 . While further research is needed to understand the best management approach in a patient with CTD-ILD and SARS-CoV-2 infection, background immunomodulatory with MMF/MPA therapy may protect patients from CSS.

Linh Truong ${ }^{1}, \mathrm{MD}$

Lila Pourzand ${ }^{2}, \mathrm{MD}$, Assistant Professor

Elizabeth R. Volkmann ${ }^{1}$ (D), MD, MS, Assistant Professor

${ }^{1}$ Department of Medicine, University of California, Los Angeles, David Geffen School of Medicine;

${ }^{2}$ Department of Radiology, University of California, Los Angeles, David Geffen School of Medicine, Los Angeles, USA.

This work was supported by grants from the NHLBI/NIH: K23

HL150237-01 (ERV) and the Rheumatology Research Foundation (ERV). ERV reports personal fees from Boerhinger Ingelheim, Forbius, and grants from Forbius and Corbus.

Address correspondence to Dr. E.R. Volkmann, 1000 Veteran

Avenue, Ste 32-59, Los Angeles, CA 90095, USA.

Email: evolkmann@mednet.ucla.edu.

\section{ACKNOWLEDGMENT}

We thank the patients for allowing us to present their cases in this manuscript.

\section{REFERENCES}

1. Weiss P, Murdoch DR. Clinical course and mortality risk of severe COVID-19. Lancet 2020;395:1014-5.

2. Wu C, Chen X, Cai Y, Xai J, Zhou X, Xu S, et al. Risk factors associated with acute respiratory distress syndrome and death in patients with coronavirus disease 2019 pneumonia in Wuhan, China. JAMA Intern Med 2020;180:934-43.

3. de Lathouder S, Gerards AH, de Groot ER, Valkhoff M, Aarden LA. Mycophenolic acid and methotrexate inhibit cytokine production via different mechanisms. Eur Cytokine Netw 2002;13:317-23. 
4. Mihai C, Dobrota R, Schroder M, Garaiman A, Jordan S, Becker $\mathrm{MO}$, et al. Covid-19 in a patient with systemic sclerosis treated with tocilizumab for SSc-ILD. Ann Rheum Dis 2020;79:668-9.
5. Huang $\mathrm{H}$, Zhang M, Chen C, Zhang H, Weit Y, Tian J, et al. Clinical characteristics of Covid-19 in patients with pre-existing ILD: a retrospective study in a single center in Wuhan, China. J Med Virol 2020 (E-pub ahead of print). 